\title{
FDS+Evac: Herding Behavior and Exit Selection
}

\author{
TIMO KORHONEN ${ }^{1}$, and SIMO HELIÖVAARA ${ }^{2}$ \\ ${ }^{1} \mathrm{VTT}$ Technical Research Centre of Finland \\ P.O. Box 1000, FI-02044 VTT, Finland \\ ${ }^{2}$ Systems Analysis Laboratory \\ Aalto University \\ P.O. Box 11100, FI-00076 Aalto, Finland
}

\begin{abstract}
The behavior of the occupants is one major factor determining the outcome of building evacuations. Two different occupants may make very different decisions in similar situations. Evacuation simulation models should consider this factor and enable simulations with different behavioral scenarios. We present how three different behavioral types have been implemented in the agent based evacuation model FDS+Evac. The considered types are 'conservative', 'active', and 'herding' agents and they differ in the way they select their target exits. Conservative agents prefer familiar routes, active agents actively observe their environment to find the fastest exit route, and herding agents tend to follow others. The factors affecting the exit selections include the distance to the exit, the behavior of the other agents, visibility and familiarity of the exits, and the smokiness along the exit routes. Some verification simulations are made and the presented model is seen to work as intended. A larger scale evacuation scenario is analyzed to illustrate the effect of the agent types on the outcome of evacuations. The analysis shows that the presence of active agents decreases the average egress time even if there is a large number of herding agents present. Active agents discover the less used, faster exit routes, like emergency exits, and lead the following herding agents also to these routes. This results in more even exit usage and speeds up the whole evacuation.
\end{abstract}

KEYWORDS: modeling, egress, exit selection, human behavior, herding, FDS+Evac.

\section{INTRODUCTION}

In emergency evacuations, occupants' behavior in given situations may vary largely depending on their individual characteristics. One key factor is the available information; all evacuees may not know all possible exit routes, and thus may end up using inferior options. Also, even if everybody were aware of all possible exits, individual characteristics may lead them to selecting different routes. Some people may prefer exit routes that they are familiar with even if faster options were apparent $[1,2]$.

A common observation from real life evacuations has been that many occupants tend to select the exit where the majority of the others are heading. Such behavior seems to occur even if shorter and faster routes were available and clearly visible [3-5]. This behavior is usually called herding. Many prescriptive fire codes implicitly assume that the total exit width of buildings is used in egress. Herding behavior, as well as people's tendency to favor the familiar routes, may easily lead to outcomes that contradict with these assumptions. There are many studies and models about the herding of pedestrians $[3,4,6]$, but large interest has also been given to such behavior in animal populations, see e.g. Ref. [7]. Some recent results also show that the characteristics of herding are rather similar with both people and animals $[8,9]$.

Recent observations on different egress situations [5] give many examples where the exit usage during egress is not optimal. In almost all cases studied in that work, occupants used the exits of the buildings inefficiently. In some cases just one leaf of double leaf doors was used even though the other leaf could be opened just by pushing it. People decide to follow others using the part of the door that had the leaf already opened or held open by someone in front. This was seen to happen also when there was congestion in front of the doors, i.e., people had to slow down and wait a little bit to go through the door that had the leaf already opened. A related example is a fire drill in a vocational high school that had a staircase ending at ground level, where the usual way out was through the entrance hall. But there was also an emergency exit on a side wall at the bottom of the stairs. During the fire drill, it was observed that it took two and a half minutes before the emergency exit was opened, apparently by a safety organization member, and the evacuees started to use this way out. Just one person was enough to trigger the use of an emergency exit. 
The impact of individual differences, like tendencies to favor familiar routes or to follow others, should be taken into account in evacuation simulation models. The occupants of an office building may have a different distribution of behavioral types than the people in a shopping mall or at a rock concert. Hence, when simulating different evacuation scenarios, it should also be interesting to study how the distribution of characteristics in the population may affect the outcome. The modeling of different agent types has been considered in many previous studies. Many times the differences relate to the physical properties of the agents, e.g., the body size and walking speed may differ between males, females, and children [10,11]. Also agents with different behavioral types have been considered, for instance, the different characteristics of staff members and regular crowd members [12] or senior and junior occupants [13].

In this article, we present how different behavioral types have been added to the FDS+Evac evacuation model [10]. Three different agent types are considered: a conservative type, an active type, and a herding type. We describe the characteristics of the types and present the results of different verification simulations. We also study how the proportions of different agent types in the population affect the outcome of an evacuation scenario. Also presented is how the exit selection model can be implemented using a computationally efficient and easily programmable penalty function formulation.

\section{MODEL}

FDS+Evac [10] is an agent-based egress calculation module that is designed to work on the platform of Fire Dynamics Simulator (FDS) [14]. FDS is a fire simulation software program and the evacuation simulations of FDS+Evac can be fully coupled with the fire simulations. Pedestrians are modeled as individual agents, which are moving in horizontal planes representing the floors of buildings. The trajectories of the agents are found by solving a coupled differential equation system consisting of equations of motion for each agent. Thus, the model can be categorized to be a continuous time and space type egress model. This approach allows each agent to have its own individual properties and behavioral models. The body diameters, motive force strengths, and walking speeds are, by default, drawn randomly for each generated agent from uniform distributions. There are also small random fluctuation forces and torques and there is some randomness present in the behavioral models also. The detection and reaction times of the agents are drawn from the user given statistical distributions. Thus, FDS+Evac can also be considered to be a stochastic egress model.

The model of Helbing et al. $[3,15,16]$ is used in FDS+Evac for the movement of the agents, their physical interactions, and their tendency to keep some distance to the other agents. One advantage of this is that the behavior of each agent can be altered by adjusting its individual preferred moving velocity, which describes the speed and direction in which the agent attempts to move. For a detailed description of the model, see the original references. The original model of Helbing et al. uses one circle to represent each agent, but FDS+Evac uses a three-circle model [10,17], which represents the elliptical cross section of a human body better and also enables the modeling of body rotation. The original agent movement model introduced by Helbing et al. and its three circle equivalent are not well suited to situations where agents are going in different directions and their paths are crossing or opposite to each other. To overcome this deficiency, FDS+Evac uses a counter flow model presented by Heliövaara and Korhonen [18].

An agent aims directly towards the chosen target exit if there are no obstacles blocking direct movement and the local agent density is not too high. In other cases, it is guided to the chosen exit by a preferred walking direction vector field, which is obtained using FDS and its flow solver. This vector field is obtained as a solution to a flow problem of a two-dimensional incompressible fluid, where the chosen exit acts as a fan, which extracts fluid out of the domain. This method, or rather a trick, produces a nice directional field for egress towards the chosen exit. The actual path of the agent will deviate from this fictive flow field or direct aiming due to the interactions with the other agents and walls and due to inertia.

The exit selection algorithm of FDS+Evac is based on a theoretical game model described and analyzed in detail in Refs. $[10,19,20]$. The agents observe the actions of the others and select the target exit through which the evacuation is estimated to be the fastest. The evacuation time of each agent to each exit is calculated from the distances to the exits and the congestion in front of the exits. The estimated evacuation time is not the only criterion considered in the model; also the visibility of the exits and the fire related conditions at the exits affect the decision, as well as the familiarity with the different exits, which can be defined for each agent by the user. These three criteria define five different exit groups as presented in 
Table 1. An agent regards a visible exit as a 'no smoke' exit if there is less smoke than a user given amount along the direct line to the exit. For non-visible exits the smoke concentration at the position of the agent is used to estimate the presence of smoke or not. If there is no sign of smoke then it is assumed that there is no reason for the agents to prefer other routes than the familiar ones. If there are clues of smoke then the agents are more willing to use visible exits, familiar or not, than non-visible ones to get out safely. The agents select an exit with the smallest preference number, and only if two or more exits share the smallest preference, the decision between these is made by minimizing the estimated evacuation time. Previously, all agents of FDS+Evac selected their target exits using this algorithm. In this paper, we present two new agent types besides this original type. The original type agents are from now on called 'conservative' agents.

Table 1. Preference order of the exits.

\begin{tabular}{|c|c|c|c|}
\hline Preference number & Visible & Familiar & No smoke \\
\hline 1 & yes & yes & yes \\
\hline 2 & no & yes & yes \\
\hline 3 & yes & no & yes \\
\hline 4 & yes & yes/no & no \\
\hline 5 & no & yes & no \\
\hline
\end{tabular}

The first new agent type is 'active' agents, who use a different preference order for the exits than the 'conservative' type. Active agents regard all visible exits also to be in the same preference group as familiar exits. The second new agent type is 'herding' agents. Herding agents use only the familiar exits, if any are given by the user. If there is no familiar exit at the current floor of the building then a herding agent looks at its nearest neighbor agents and tries to follow them.

\section{Conservative Agents}

The conservative agent type in the present version of FDS+Evac (v2.3.0 onwards) is modified from the older versions (prior to v2.3.0). If there is some smoke in the exit routes then the preference order is modified a little bit. All visible exits are put to the same preference group regardless of their familiarity when there is smoke on the exit routes. It is assumed that the agents have higher stress and are willing to change their behavior such that any exit that leads out of the smoke is used. The other modification in the present program version is that the $\mathrm{L} 1$ distance is used to describe the walking distance to the non-visible exits and the L2 distance is used only for the visible ones. The L2 distance is the straight Euclidean distance between two points, while L1 distance is the sum of absolute differences in the coordinates of the points. The L1 distance is also called the Manhattan metric. The name alludes to the grid layout of the streets of Manhattan, which causes the shortest path between two points to be equal to the L1 distance. We use the Manhattan metric to approximate the walking distance to non-visible exits because corridors and obstacles often make buildings resemble grid geometries. This approximation is used because the current version of FDS+Evac does not include calculation of the actual shortest path. While the shortest path calculation is likely to be implemented in the future, the L1 distance gives rather accurate approximations in most building geometries.

The movement of the conservative agent type is triggered by the user given detection time and reaction time distributions. The detection time can be shorter than the user given distribution if there is enough smoke to trigger the detection. The smoke can be detected either by the local smoke concentration at the position of the agent (detection by senses) or detection by a device (usually a smoke or a heat detector). If a conservative agent gets lost then it starts to behave like a herding agent for a while until it is able to locate an exit by its own reasoning. An agent is lost when it cannot see any exit and does not have any familiar exit in that part of the building. This might happen if an agent is using some visible but not familiar internal door to go to some other floor or connected space within the building. It might also happen that some of the familiar routes are blocked by smoke and the agent ends up using an unfamiliar route. Conservative type agents could be used in many building evacuation situations, e.g., the customers in a shopping mall, who know the main exits but are not so willing to use special emergency exits. 


\section{Active Agents}

The active agent type is very similar to the conservative agent type. The difference is that active agents observe their environment actively to find the fastest exit route. Hence, active agents prefer all visible exits similarly regardless of whether the exits are familiar or not. Detection and reaction times are like those of conservative agents and lost active agents are treated similarly to lost conservative agents. Heterogeneous crowds contain more and less observant occupants. The less observant will head to their familiar exits or follow others, while the more observant ones actively look for possible faster egress routes. The active agent type can be used to model these more observant crowd members. The existence of active agents may be very significant to the outcome of evacuations as, in addition to themselves, they may also lead herding agents to some normally unused exits. This kind of behavior was observed quite often in the evacuation drills and real fire alarms in Finland analyzed by Rinne et al. [5] as discussed in the introduction.

\section{Herding Agents}

The second new agent type is 'herding' agents. These are assumed to be unfamiliar with the geometry and they will not use any exit, unless it is regarded as a familiar exit. Usually just the doors that they used to enter the building could be regarded as familiar exits. This agent type represents also lost agents, who can not figure out any available exit. Herding agents are looking around and seeing what the other agents are doing and, if some of the others are heading towards an exit, they start to follow these other agents. A herding agent will remain still even after detecting the fire and its reaction time has passed if it has not been able to select any exit either by being familiar with it or by observing other agents. If the nearest neighbors of a herding agent are heading towards some exit then the herding agent starts to follow these agents almost immediately regardless of its detection and reaction times. If familiar exits are available at a given floor for a herding agent then it behaves like a conservative agent, i.e., the familiar route behavior overrides the herding behavior.

A herding agent always looks around and checks where its nearest neighbors are heading. By default five nearest neighbors that are within a radius of 5 meters are included. The herding agent will follow the majority of its nearest neighbors. Only those nearest neighbors are considered that are heading away from the herding agent (cosine of the angle less than -0.2). This mimics the fact that the herding agent wants to follow other agents, not to lead them. The default distance is $5 \mathrm{~m}$ but the user may change this if it is not appropriate for the application in question. The default value was selected after some test simulations as it was seen to operate reasonably well for the verification cases. In principle, the distance should depend on the agent density and the typical room size of the building. But it was seen that this distance had no effect with reasonable design densities, because most of the agents have at least one neighbor within a $5 \mathrm{~m}$ distance.

If a herding agent has not been able to get any exit information from its nearest neighbors nor has it any familiar exit route available, then it looks for all visible agents and tries to find the nearest agent that is heading to some exit. It constantly updates this information as long as there are some visible agents heading towards exits. Note here also that the walking directions of the other agents have a similar effect as the effect of the moving directions of the nearest neighbors: If the other agent is heading towards the herding agent the direction is recorded when the moving agent has passed the herding agent. If all moving agents have disappeared then the herding agent will remember where the last visible moving agent went. If the herding agent has detected the fire and the reaction time has passed then the agent starts to move towards this previously recorded exit direction.

If an agent is not able to find an exit after entering a floor through a door, it starts the herding behavior by checking the nearest neighbors and other visible agents. If the agent is not able to find any information of exits, it moves away from the entry door a couple of meters, stops there, and continues to observe other agents. At this point, the herding agent has already started to evacuate the building so the detection times and reaction times are not used anymore, i.e., the agent will continue to move immediately when it gets information on a new target exit to be used.

The herding behavior could be easily modified to take into account effects like patrons vs. staff, children vs. adults, etc., where some agents are more likely to be followed than others. This could be modeled by giving different weights to the influence of different agent types. For now, the only effect that the user can specify is the distance dependence of the effect of the nearest neighbors on the decision of the herding 
agent. The user can specify that the nearer neighbors have larger weights than the more distant ones using a linear function.

\section{Implementation of Penalty Functions}

The presented two-phase exit selection algorithm is implemented in FDS+Evac using a penalty function formulation, where a preference-dependent penalty term is added to the estimated egress time of each exit. The penalty term is defined as a product $P_{i}(k) M$, where $P_{i}(k)$ refers to the preference number (see Table 1 ) of exit $k$ to agent $i$ and $M$ is a sufficiently large constant. Using these notations, agent $i$ selects the exit $k$, solving the following optimization problem:

$\arg \min _{k \in K} T_{i}(k)+P_{i}(k) M$,

where $T_{i}(k)$ is the estimated evacuation time of agent $i$ through exit $k$, which is calculated from the distance to the exit and the queue in front of the exit. $K$ denotes the set of all exits. The constant $M$ is the amount of penalty assigned to an exit when its preference number $P_{i}(k)$ increases by one. When $M$ is sufficiently large, the value of $T_{i}(k)$ is always smaller than $M$, and an exit with the smallest possible value of $P_{i}(k)$ is selected. Hence, the estimated evacuation time $T_{i}(k)$ only plays a role when comparing exits with the same preference numbers, and thus, the minimization of Eq. 1 is equivalent to the presented two-phase model. Different agent types can be easily considered by altering the preference numbers, $P_{i}(k)$, of the different types of exits.

The formulation of the penalty function is computationally efficient and flexible for further modifications. The algorithm can be easily altered to use an arbitrary preference order and new criteria can be easily added. If the algorithm was programmed using multiple nested if-clauses, all changes would require a bigger programming effort.

\section{SIMULATION RESULTS}

To test the proposed model for herding behavior, four different test cases were considered. The first three cases use basically the same geometry but they verify different aspects of the model. The fourth test case is a much larger and more complex geometry, which is used to see how the exit selection algorithm works with the herding algorithm.

\section{Verification Case 1}

The first test case is the same geometry as used by Gwynne et al. [13] to introduce social adaptation within the buildingExodus evacuation model [21]. The geometry and the initial positions of the agents are shown in Fig. 1. It is a $20 \mathrm{~m}$ long and $5 \mathrm{~m}$ wide space and there are four 'seniors' (large black ellipses) at the back end of the room and twenty 'juniors' (small light gray ellipses) closer to the $1.5 \mathrm{~m}$ wide exit located at the right. The juniors are modeled as herding agents and seniors as conservative agents. The thin black lines mark the areas where the agents are randomly generated. FDS+Evac is a stochastic evacuation program: The initial positions of the agents are random, the personal characteristics of the agents are drawn from statistical distributions, and there is some stochastic behavior in the algorithms, like small random forces. Thus, the outcome of two FDS+Evac simulations with the same user input will give different results and for each scenario many simulations should be performed in order to obtain statistical significance. The herding model presented in this paper differs somewhat from the social adaptation model but it is seen to perform similarly: the senior members (modeled as active agents) are guiding the junior members (herding agents) towards the exit as the seniors pass by the junior crowd, see Fig. 2. The average emptying time of the room was $35 \pm 2 \mathrm{~s}$, where the error is the standard deviation of the 50 simulations performed. The default 'Adult' type from FDS+Evac was used for 'seniors' and 'Child' type for 'juniors'. The properties of the 'Adult' and 'Child' types are described in Ref. [10]. 


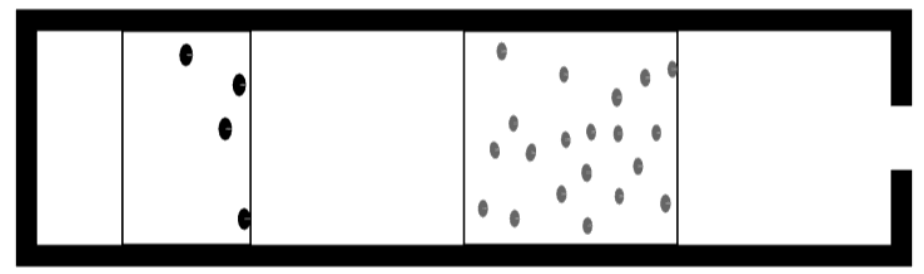

Fig. 1. The initial positions of the agents in the verification Case 1.

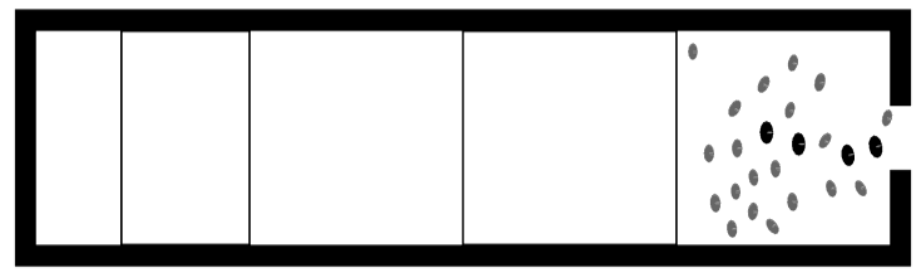

Fig. 2. Snapshot from a verification Case 1 simulation.

\section{Verification Case 2}

This verification case has the same geometry as Case 1, but there are now two $1.5 \mathrm{~m}$ wide exits at the right end of the room. The geometry and the initial positions of the agents are shown in Fig. 3. The two large black agents are heading to the upper exit and the two large dark gray ones marked with circles to the lower exit. There are twenty small light gray agents that are herding agents. It is expected that the herding agents start to follow the black and dark gray agents towards both exits, but the proportion ending up at upper and lower exit vary between different simulations. As the simulation proceeds the herding agents start to follow the majority of their nearest neighbors, see Fig. 4, where the herding agents are shown as small black (upper exit), dark gray with circles (lower exit), or light gray (no exit yet) ellipses according to the chosen exit. The lower exit is used by 9.4 agents on the average with standard deviation of 7.8 agents, when 50 simulations were done. The standard deviation is quite large so that the random initial positions of the agents and the stochastic nature of FDS+Evac made the herding agents sometimes mainly to follow agents going towards one of the exits. The average emptying time of the room was $45.4 \mathrm{~s}$ with $3.6 \mathrm{~s}$ standard deviation. The emptying time is ten seconds longer than in the case where there is just one exit. It can be seen from the simulations that there is no congestion at the two exits in Case 2 so in this case the emptying time depends on how fast the agents are able to get to the exits. The agents heading to the top exit need to avoid the ones going to the bottom one and vice versa, which reduces the speed of the agents. Also the exit usage is not at all optimal in Case 2. Depending on the initial positions of the 'senior' agents, the majority of the herding agents may end up using just one of the two available exits.

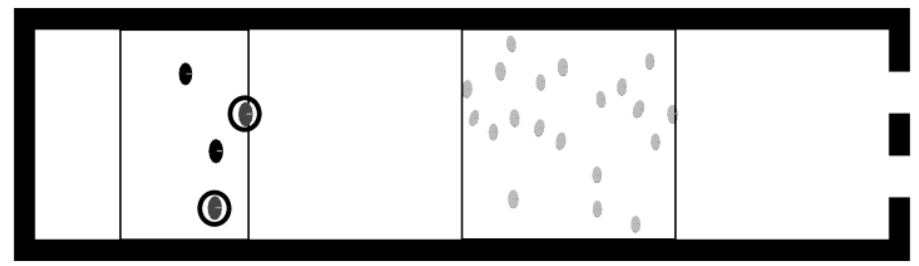

Fig. 3. Initial positions of the agents in the verification Case 2. 


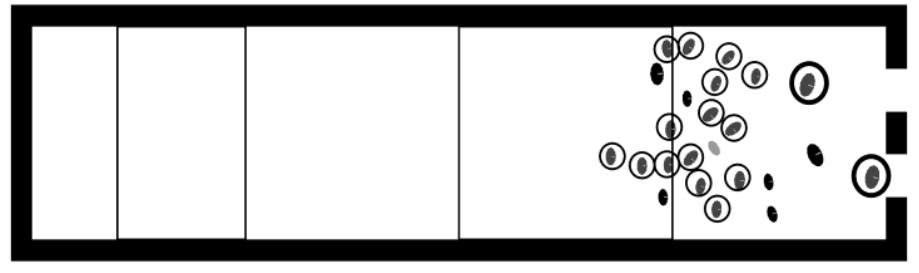

Fig. 4. Snapshot from a verification Case 2 simulation.

\section{Verification Case 3}

The third verification case has the same geometry as the previous cases, but there are now two $1.5 \mathrm{~m}$ wide exits located at the opposite ends of the room. The geometry and the initial positions of the agents are shown in Fig. 5. The black agents are heading to the right exit and the dark gray ones marked with circles to the left exit. The light gray agents are herding agents that have not yet chosen an exit. As the simulation proceeds, the herding agents start to follow the majority of their nearest neighbors, see Fig. 6 . In the simulations there were fifty 'junior' agents and eight 'senior' agents. The target exits of the eight senior agents were varied. For each scenario, one hundred simulations were performed. The results of the simulations are given in Table 2, where the averages and standard deviations of the emptying times are given together with the percentage of the junior agents that ended up using the left exit. For the scenarios, where there were two or six senior agents, all juniors were mainly following the majority of seniors producing quite skewed distributions for the exit usage.

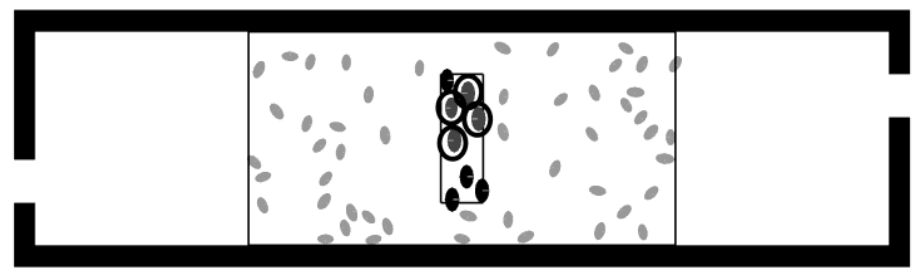

Fig. 5. Initial positions of the agents in the verification Case 3.
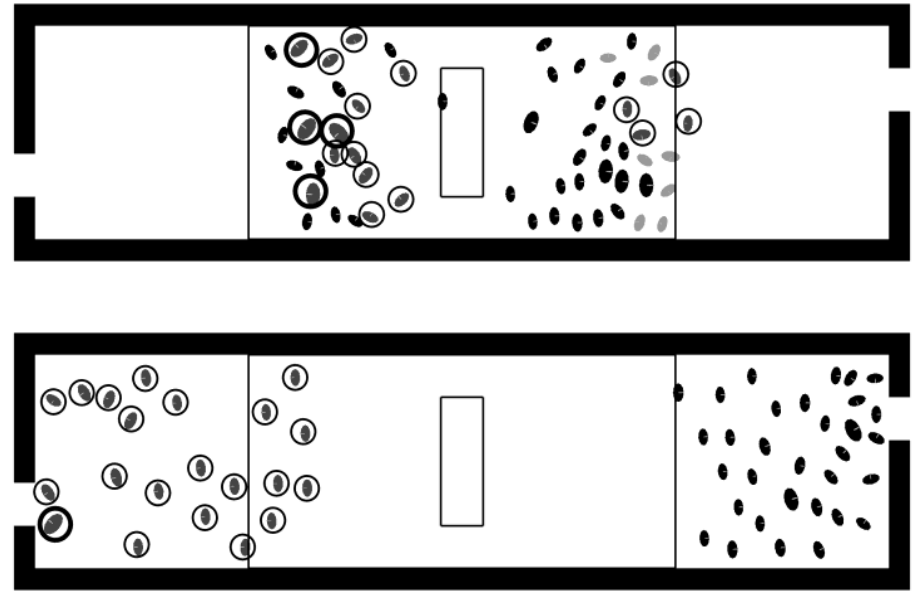

Fig. 6. Snapshots from a verification Case 3 simulation. 
Table 2. Results of the verification Case 3.

\begin{tabular}{|c|c|c|c|}
\hline \multicolumn{2}{|c|}{ Senior agents } & \multirow{2}{*}{$\begin{array}{c}\text { Time } \\
\text { (s) }\end{array}$} & $\begin{array}{c}\text { Junior agents } \\
\text { at left exit }\end{array}$ \\
\hline Left & Right & $67 \pm 11$ & $29 \pm 29 \%$ \\
\hline 2 & 6 & $65 \pm 12$ & $61 \pm 30 \%$ \\
\hline 4 & 4 & $70 \pm 10$ & $86 \pm 22 \%$ \\
\hline 6 & 2 &
\end{tabular}

\section{Verification Case 4}

To test the proposed herding and exit selection model in a larger and more complex scenario, the geometry shown in Fig. 7 was used, where there are four exits (left, top, right, bottom), each $1.25 \mathrm{~m}$ wide, and there are 400 agents, of which some portion are herding agents, some conservative agents, and some active agents. All the agents were generated using the default 'Adult' agent type of FDS+Evac. The active and conservative agents started to move towards the exits right away and the herding agents had a $60 \mathrm{~s}$ delay, if their nearest neighbors within $5 \mathrm{~m}$ were not moving. Figure 8 shows snapshots from a simulation where there are 10 active agents (marked using circles) and 390 herding agents. The initial positions of the agents are shown in Fig. 7. It can be seen that the active agents are able to guide the herding agents towards the exits as it is expected.

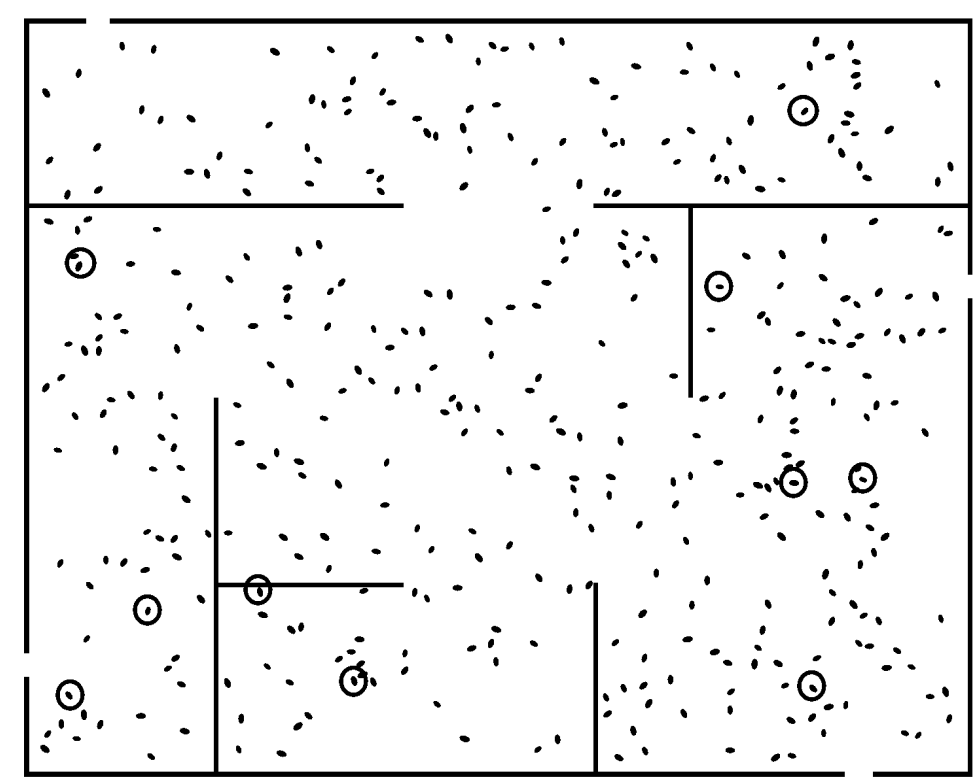

Fig. 7. Initial positions of the agents in the test Case 4. The active agents are marked with circles.

First, the difference between conservative and active agents was examined by setting the top and bottom exits as familiar exits, which are preferred by the conservative agents. Five different scenarios with $100 \%$, $75 \%, 50 \%, 25 \%$, and $0 \%$ of the agents being active agents and the rest being conservative agents were simulated. In total there were 400 agents in the simulations and for each scenario one hundred simulations were performed. The results of these five cases are shown as the top rows of Table 3, where the averages of the total evacuation times with standard deviations for the one hundred simulations are given. The usage of the different exits is also given for top and bottom exits and a combined usage for the left and right exits. The results for the evacuation times are more or less as expected, with the use of all four exits being about twice as fast as just using two exits. The usage of the top exit stayed about at $27 \%$ in every case due to the geometry and the fact that the visible (familiar) exits are preferred over the non-visible ones, so the agents in the top room use the top exit and the agents in the other rooms some other exit. 

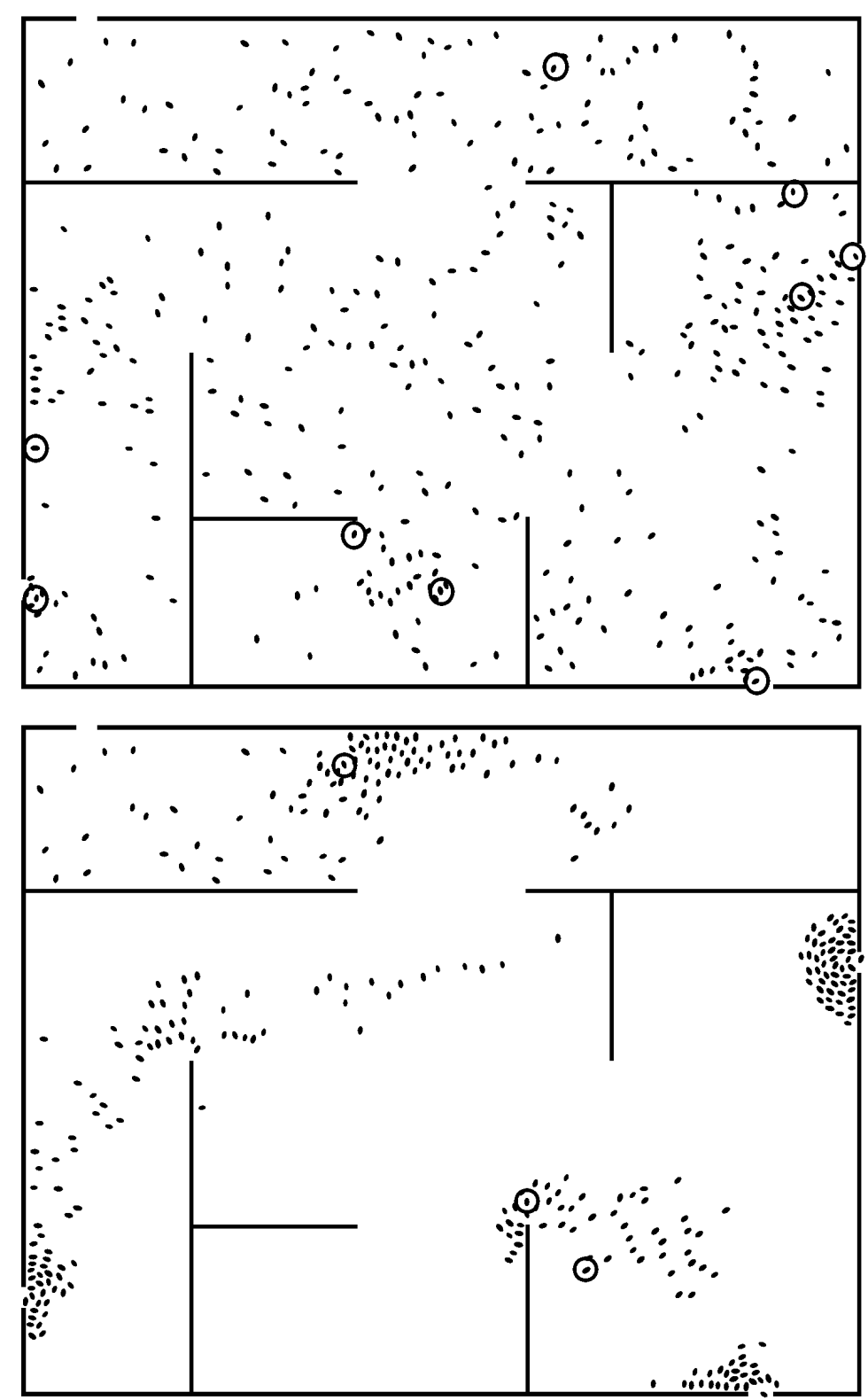

Fig. 8. Snapshots from a verification Case 4 simulation. The active agents are marked with circles.

To test the herding behavior, simulations were performed where the majority of the agents were herding type (87.5-97.5\%) and the minority were active and/or conservative type agents. The results of these simulations are summarized in Table 3. All simulations had 400 agents and for each case one hundred simulations were performed. The averages and the standard deviations of the evacuation times for different population scenarios are shown and also the average usage of the different exits. The total evacuation times shown in the table are about the same for the scenario with $100 \%$ of conservative agents and the scenarios where there were just some conservative agents and a vast majority of herding agents. This is more or less as expected, since only two exits were used and queues were formed at the exits. Even if there are only a few conservative agents to trigger the movement of the herding agents, the last herding agents are reaching the exits before the queues have cleared. For the cases where there are some active agents present, the evacuation times are faster, which is due to the fact that more exits are used to exit the building. But the times are not as fast as when there are just active agents present, because the herding agents following their neighbors do not optimize their exit choice using best response dynamics like the active agents. These findings support the fact that the presence of personnel, fire wardens and other authority figures are 
supposed to make the egress process more effective. Also frequent fire drills and practical design solutions for buildings, where many different routes to enter and to leave a building are used routinely, would be beneficial for a fast egress due to the more even use of the capacity of the egress routes.

Table 3. Results of the verification Case 4.

\begin{tabular}{|c|c|c|c|c|c|c|}
\hline \multicolumn{3}{|c|}{ Agent population } & \multirow{2}{*}{$\begin{array}{c}\text { Time } \\
\text { (s) }\end{array}$} & \multirow{2}{*}{ Top } & \multirow{2}{*}{ Bottom } & \multirow{2}{*}{$\begin{array}{l}\text { Left+ } \\
\text { Right }\end{array}$} \\
\hline Active & Conservative & Herding & & & & \\
\hline 400 & 0 & 0 & $75 \pm 3$ & $26 \%$ & $31 \%$ & $43 \%$ \\
\hline 300 & 100 & 0 & $82 \pm 3$ & $27 \%$ & $35 \%$ & $38 \%$ \\
\hline 200 & 200 & 0 & $99 \pm 4$ & $27 \%$ & $43 \%$ & $30 \%$ \\
\hline 100 & 300 & 0 & $128 \pm 5$ & $27 \%$ & $57 \%$ & $16 \%$ \\
\hline 0 & 400 & 0 & $160 \pm 5$ & $27 \%$ & $73 \%$ & $0 \%$ \\
\hline 0 & 10 & 390 & $164 \pm 22$ & $32 \%$ & $68 \%$ & $0 \%$ \\
\hline 0 & 20 & 380 & $159 \pm 17$ & $32 \%$ & $68 \%$ & $0 \%$ \\
\hline 0 & 50 & 350 & $159 \pm 12$ & $30 \%$ & $70 \%$ & $0 \%$ \\
\hline 10 & 0 & 390 & $125 \pm 21$ & $38 \%$ & $37 \%$ & $35 \%$ \\
\hline 20 & 0 & 380 & $109 \pm 15$ & $27 \%$ & $41 \%$ & $32 \%$ \\
\hline 50 & 0 & 350 & $94 \pm 10$ & $27 \%$ & $36 \%$ & $37 \%$ \\
\hline 25 & 25 & 350 & $117 \pm 14$ & $29 \%$ & $50 \%$ & $21 \%$ \\
\hline
\end{tabular}

\section{CONCLUSIONS}

Observations of real-life evacuations and fire drills show that herding behavior, where occupants follow the majority of the others, is a common phenomenon. The occurrence of herding may vary strongly between evacuations; in some cases it can be the most dominant factor affecting the exit selection while in others it may have a much smaller role. Hence, when analyzing different evacuation scenarios, a key factor for the outcome may also be the distribution of different agent types.

This article has presented three different agent types that can be used in FDS+Evac egress simulations. In addition to the conservative agent type, whose exit selection model was presented in Ref. [19], we present an active agent type and a herding agent type. The difference between these types is in the criteria, according to which they select their target exits. The conservative agents tend to favor familiar exit routes but will head to any visible exit if no familiar routes are available. The active type is not bound to the familiar exits, but will select the fastest visible exit route regardless of its familiarity. The herding agents observe the other agents instead of the available exits and head to the same exit as the majority of the agents surrounding them.

Verification simulations show that the presented model works as intended. We also ran test simulations of an evacuation scenario with 400 occupants while varying the proportions of different agent types. The results show that the existence of active agents leads to more comprehensive use of all exits, and thus decreases the average egress times. The presence of herding agents does not necessarily increase the egress times. They usually end up following the conservative or active agents causing quite similar outcomes with the cases where all agents are conservative or active. Nevertheless, the difference is that the herding agents do not try to select the fastest exit like the conservative and active ones. Hence, they do not switch to another visible exit even if there is heavy congestion at their target exit. In some situations, this herding behavior may lead to uneven usage of exits and increase the egress times dramatically. This outcome of herding has also been observed in real evacuations and evacuation drills, see Ref. [5]. For example, in many cases just one leaf of double leaf doors was used even though the other leaf could be opened just by pushing it. In another case, it took two and a half minutes before an emergency exit was opened at the bottom of a staircase, apparently by a safety organization member, and the evacuees started to use this way out. The presence of staff, floor wardens, etc. and/or frequent fire drills have definitely an effect on the egress process and these types of effects should also be considered in the simulation of emergency egress situations. 


\section{ACKNOWLEDGEMENTS}

This research was supported by the Academy of Finland.

\section{REFERENCES}

[1] Proulx, G., (1993) A Stress Model for People Facing a Fire, Journal of Environmental Psychology 13(2): 137-147. http://dx.doi.org/10.1016/S0272-4944(05)80146-X

[2] Sime, J.D., "The Concept of Panic," Fires and Human Behavior, Canter, D. (ed.), John Wiley \& Sons, 1980, pp. 63-81.

[3] Helbing, D., Farkas, I.J., Molnar, P., and Vicsek, T., "Simulation of Pedestrian Crowds in Normal and Evacuation Situations," Pedestrian and Evacuation Dynamics, Schreckenberg, M. and Sharma, S.D. (eds.), Springer, 2002, pp. 21-58.

[4] Pan, X., "Computational Modeling of Human and Social Behaviors for Emergency Egress Analysis," Dissertation, Stanford University, Stanford, CA, USA, 2006.

[5] Rinne, T., Tillander, K., and Grönberg, P., "Data Collection and Analysis of Evacuation Situations," VTT Research Notes 2562, VTT Technical Research Centre of Finland, Espoo, Finland, 2010.

[6] Low, D., (2000) Following the Crowd, Nature 407: 465-466. http://dx.doi.org/10.1038/35035192

[7] Couzin, I.D., Krause, J., Franks, N.R., and Levin, S.A., (2005) Effective Leadership and DecisionMaking in Animal Groups on the Move, Nature 433: 513-516. http://dx.doi.org/10.1038/nature03236

[8] Dyer, J.R.G, Ioannou, C.C., Morrell, L.J., Croft, D.P., Couzin, I.D., Waters, D.A., and Krause, J., (2008) Consensus Decision Making in Human Crowds, Animal Behaviour 75: 461-470. http://dx.doi.org/10.1016/j.anbehav.2007.05.010

[9] Dyer, J.R.G, Johanson, A., Helbing, D., Couzin, I.D., and Krause, J., (2009) Leadership, Consensus Decision Making and Collective Behavior in Humans, Philosophical Transactions of the Royal Society B: Biological Sciences 364: 781-789. http://dx.doi.org/10.1098/rstb.2008.0233

[10] Korhonen, T. and Hostikka, S., "Fire Dynamics Simulator with Evacuation: FDS+Evac Technical Reference and User's Guide," VTT Working Papers 119, VTT Technical Research Centre of Finland, Espoo, Finland, 2009.

[11] "Simulex User Guide - Virtual Environment 5.8," Integrated Environmental Solutions Ltd., Glasgow, Scotland, UK, 2009.

[12] Pelechano, N., O’Brien, K., Silverman, B., and Badler, N., "Crowd Simulation Incorporating Agent Psychological Models, Roles and Communication," First International Workshop in Crowd Simulation (V-CROWDS 05), Lausanne, Switzerland, 2005, pp. 21-30.

[13] Gwynne, S., Galea, E.R., and Lawrence, P.J., (2006) The Introduction of Social Adaptation within Evacuation Modelling, Fire and Materials 30: 285-309. http://dx.doi.org/10.1002/fam.913

[14] McGrattan, K.B., Hostikka, S., and Floyd, J.E., "Fire Dynamics Simulator (Version 5), User's Guide," NIST Special Publication 1017-1, National Institute of Standards and Technology, Gaithersburg, MD, USA, 2007.

[15] Helbing, D. and Molnár, P., (1995) Social Force Model for Pedestrian Dynamics, Physical Review E 51: 4282-4286. http://dx.doi.org/10.1103/PhysRevE.51.4282

[16] Helbing, D., Farkas, I., and Vicsek, T., (2000) Simulating Dynamical Features of Escape Panic, Nature 407: 487-490. http://dx.doi.org/10.1038/35035023

[17] Langston, P.A., Masling, R., and Asmar, B.N., (2006) Crowd Dynamics Discrete Element MultiCircle Model, Safety Science 44: 395-417. http://dx.doi.org/10.1016/j.ssci.2005.11.007 
[18] Heliövaara, S. and Korhonen T., "Counterflow Model for FDS+Evac," Proceedings of the $5^{\text {th }}$ International Conference on Pedestrian and Evacuation Dynamics, March 8-10, 2010, Gaithersburg, MD, USA, in print.

[19] Ehtamo, H., Heliövaara, S., Korhonen, T., and Hostikka, S., (2010) Game Theoretic BestResponse Dynamics for Evacuees' Exit Selection, Advances in Complex Systems, 13: 113-134. http://dx.doi.org/10.1142/S021952591000244X

[20] Heliövaara, S., Ehtamo, H., Korhonen, T., and Hostikka, S., "Modeling Evacuees' Exit Selection with Best-Response Dynamics," Pedestrian and Evacuation Dynamics 2008, Klingsch, W.W.F., Rogsch, C., Schadschneider, A., and Schreckenberg, M. (eds.), Springer, 2010, pp. 309-319.

[21] Galea, E.R., Gwynne, S., Lawrence, P.J., Filippids, L., BlackShields, D., and Cooney, D., "BuildingEXODUS User Manual, Version 3.01," CMS Press, London, U.K., 2000. 